\title{
A Simplified Fabrication Technique for Tilted Fiber Bragg Grating for The Simultaneous Measurement of Refractive Index and Temperature of Liquids
}

\author{
Akihiro KAMEYAMA $^{* 1}$, Masahito KATTO ${ }^{* 2}$ and Atsushi YOKOTANI*1 \\ ${ }^{* 1}$ Faculty of Engineeing, University of Miyazaki, 1-1 Gakuenkibanadai-Nishi, Miyazaki, Miyazaki \\ 889-2192, Japan \\ E-mail: kame@opt.miyazaki-u.ac.jp \\ ${ }^{*}$ Center of Collaborative Research and Community Cooperation, University of Miyazaki, 1-1 \\ Gakuenkibanadai-Nishi, Miyazaki, Miyazaki 889-2192, Japan
}

\begin{abstract}
We have developed a simplified technique to fabricate tilted fiber Bragg gratings (TFBGs) for simultaneous measurement of the refractive index and temperature of liquids in biochemical and medical applications. We used a combination of a $4 \omega \mathrm{Nd}$ :YAG laser and a phase mask, which is simpler than the conventional method using a $2 \omega \mathrm{cw} \mathrm{Ar}^{+}$laser and a Lloyd mirror interferometer. The peak of the cladding mode of the TFBG shifted to the longer wavelength side as the refractive index of liquid increased. Furthermore, both the peak of the cladding mode and that of the core mode shifted to the longer wavelength side as the temperature of liquid increased. We found that a refractive index from 1 to 1.41 , and a temperature from 293 to $353 \mathrm{~K}$ could be measured with a single probe of the fabricated TFBGs.
\end{abstract}

DOI: 10.2961/jlmn.2014.03.0009

Keywords: tilted fiber Bragg grating, refractive index, temperature, phase mask, core mode, cladding mode

\section{Introduction}

Fiber Bragg gratings (FBGs), which consist of a periodic modulation of the refractive index in the core of a singlemode optical fiber, are widely used for optical communication devices, temperature sensors, and distortion sensors [1]. In addition to FBG sensors, tilted fiber Bragg gratings (TFBGs) have recently been attracting considerable attention for their use in sensing applications [2-5]. In a TFBG, information in the cladding as well as in the core affects its transmission spectra. Therefore, although the conventional FBG can detect only mechanical phenomena of the core such as a change in length since the cladding prevents the optical information coming to the core from the outside, a TFBG can also detect non-mechanical phenomena since the information at the surface of the cladding affects the signal. Of course, TFBG sensors have the same advantages as FBG sensors; i.e. they are harmless, low cost, compact, and immune to electromagnetic interference.

TFBGs have conventionally been fabricated using the Lloyd mirror interferometer using a 244-nm $\mathrm{Ar}^{+}$ion laser, and the spectra were observed by Laffont and Ferdinand [6]. However, adjusting the Lloyd mirror causes an instability of the period of the grating, and furthermore, the use of an $\mathrm{Ar}^{+}$ion laser makes it difficult to consider using this technique for commercial applications.

Therefore, we have developed a simplified fabrication technique for TFBGs intended for practical applications by utilizing the commercially available pulsed Nd:YAG laser

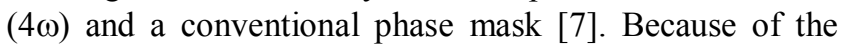
excellent spatial coherency and relatively high peak power of the Nd:YAG laser, we obtained a large space in which the periodic amplitude modulation of the laser was suitable for processing the TFBG beneath the phase mask.

One problem with the TFBG sensor is that the peak position of the cladding mode changes not only because of the refractive index but also because of the temperature. When we use TFBGs as sensors of bio-chemical phenomena in the medical and biological fields, it is necessary to measure the refractive index and temperature at the same time, because in a living body, the temperature is constantly changing. The exact refractive index cannot be found unless we know the temperature of a living body. In addition, it is necessary to fabricate a small sensor that can simultaneously measure the refractive index and the temperature with one device. Therefore we focused on the core mode of the TFBG that changes because of the temperature, but does not change because of the refractive index.

In this paper, we have characterized the TFBG fabricated using our technique as a sensor for measuring the refractive index and temperature of liquids. As a result, we have obtained promising results for simultaneous measurement of the refractive index and temperature.

\section{Experimental}

\subsection{Fabrication of TFBG}

The experimental setup used to fabricate a TFBG is shown in Fig. 1. We used a $4 \omega \mathrm{Nd}$ :YAG laser and a wavelength separator to produce a pulsed 266-nm UV laser beam to fabricate the TFBG [7]. This laser produced laser pulses with an energy of $50 \mathrm{~mJ} /$ pulse and a pulse width of 
7- ns. The beam was introduced to an optical fiber core through a phase mask made of silica glass. In this technique, a periodically modulated UV beam was produced by interference of two diffracted laser beams caused by the phase mask.

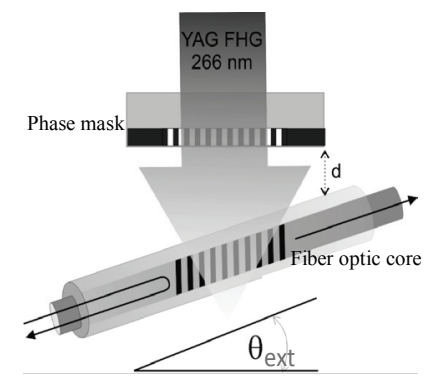

Fig. 1 Experimental setup for fabrication of TFBG.

A germanium-boron-co-doped single-mode fiber SBG15 (Newport Corp.) was used for the sample. We used 250 -mm-long sample fibers. After $\mathrm{H}_{2}$-loading, a 50-mm-long polymer jacket of the center part of the fiber was stripped to expose the fiber to UV beam irradiation. The fiber was then connected to an optical spectrum analyzer (OSA) and an amplified spontaneous emission (ASE) light source. The fiber Bragg grating was made in the center part of the fiber using a phase mask technique. A 10-mm-long phase mask with a grating period of $1.065 \mu \mathrm{m}$ was used. This phase mask creates a grating in the fiber core with a period $\Lambda_{t f b g}$ along the longitudinal direction, which is expressed as follows,

$$
\Lambda_{t f b g}=\frac{\Lambda_{p m}}{2 \cos \theta_{e x t}}
$$

where $\Lambda_{p m}$ and $\theta_{\text {ext }}$ are the grating period of the phase mask and the external tilt angle between the grating and the sample fiber, respectively.

The fiber was fixed on a rotary stage to easily adjust the $\theta_{\text {ext }}$. A $\theta_{\text {ext }}$ of $11^{\circ}$ was selected in order to obtain strong and sharp- peaks of the cladding mode [7]. Because the UV beam refracts at the interface between air and fiber material during fabrication, the tilt angles in the fiber core reach $7.3^{\circ}$. A probe light with a wavelength range from $1520 \mathrm{~nm}$ to $1610 \mathrm{~nm}$ was used for the transmission spectral measurement. Two mechanical splicers were used to connect the fiber to the light source and the OSA. The typical irradiation period of the UV beam to obtain sufficient intensity for measurement was 20 minutes. We checked the spectral change during TFBG fabrication.

\subsection{Measurement of peak shift of TFBG against refrac- tive index and temperature of liquid}

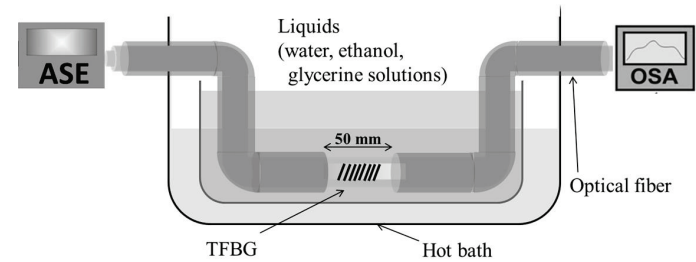

Fig. 2 Experimental setup for measuring peak shift of TFBG against refractive index and temperature of liquids.
The experimental setup to measure the peak shift of the TFBG in order to measure the refractive index and temperature of liquid is shown in Fig. 2. We immersed the fabricated TFBG in different kinds of liquids. The liquids used included water, ethanol, and various aqueous solutions of glycerine. The concentrations of the solution used in the experiment ranged from $12 \%$ to $84 \%$. We used glycerine because the refractive index of the solution can be easily adjusted in a wide index range by simply changing the ratio of glycerine and water. The refractive indices of the samples are listed in Table 1 [8]. The liquids were treated in a hot bath, and the temperature of the liquids was measured by a thermometer within an accuracy of $1 \mathrm{~K}$. The TFBG was connected to an OSA and an ASE light source while it was immersed in the liquids. We observed the transmission spectra of the core and cladding modes and investigated the relationship between the spectral change and the change in refractive index and temperature.

\section{Results}

Table 1 Refractive index of sample

\begin{tabular}{ll}
\hline \multicolumn{1}{c}{ Sample } & Ref. index $\left(20^{\circ} \mathrm{C}\right)$ \\
\hline Air & 1.0003 \\
Water & 1.3255 \\
Ethanol & 1.3539 \\
Glycerine $12 \%$ & 1.3417 \\
Glycerine $24 \%$ & 1.3579 \\
Glycerine $35 \%$ & 1.3727 \\
Glycerine $46 \%$ & 1.3876 \\
Glycerine $66 \%$ & 1.4146 \\
Glycerine $84 \%$ & 1.4389 \\
\hline
\end{tabular}

\subsection{Characteristics of peak shift against refractive index of liquid}

Figure 3 shows the transmission spectra of the $7.3^{\circ}$ TFBG after 20 minutes of processing. We observed not only the core mode, but also the cladding modes in a spectral range from approximately $1520 \mathrm{~nm}$ to $1569 \mathrm{~nm}$. The peak wavelength and the intensity of the core mode were $1572 \mathrm{~nm}$ and $-3 \mathrm{~dB}$, respectively. The maximum intensity of the cladding mode was $-8.2 \mathrm{~dB}$ at $1549 \mathrm{~nm}$. In the cladding mode, superposition of two coupling modes $\left(\mathrm{LP}_{0 \mathrm{n}}\right.$ and $\mathrm{LP}_{1 \mathrm{n}}$ ) was clearly observed [7].

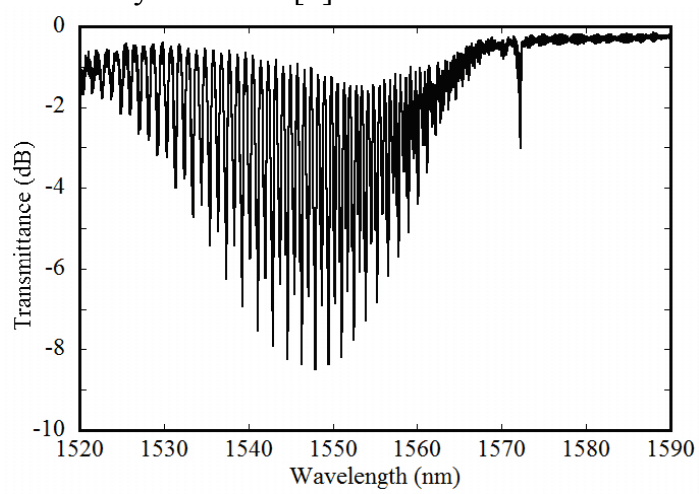

Fig. 3 Transmission spectra of fabricated TFBG with tilt angle of $7.3^{\circ}$. 
We used the $7.3^{\circ}$-TFBG to measure the peak shift as a function of the refractive index of liquids. The typical results are shown in Fig. 4a and 4b. In Fig. 4a, the spectral change of a selected peak in the cladding modes is indicated for water and for aqueous solutions of glycerine of 24 and $35 \mathrm{wt} \%$. We selected a peak of the cladding mode at $1540.68 \mathrm{~nm}$ at water. As shown in Fig. 4a, the wavelength shifted from $1540.80 \mathrm{~nm}$ to $1540.85 \mathrm{~nm}$, which corresponds to a refractive index from 1.3579 to 1.3727 . We were able to show that the spectral change was identifiable with refractive index differences of only 0.0148 from this data. The peak wavelength was at $1541.16 \mathrm{~nm}$ at aqueous solutions of glycerine of $66 \mathrm{wt} \%$, although this is not shown in the figure. No peak was observed in the case of $84 \mathrm{wt} \%$ solution, because the index was almost the same as the effective refractive index of the cladding mode. In addition to the wavelength change, a smooth change in the peak intensity was also observed.
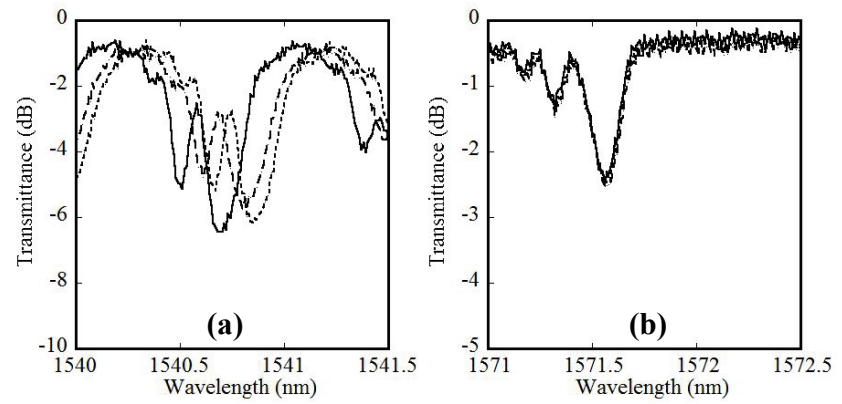

Fig. 4 Peak shift of core and cladding modes of $7.3^{\circ}$-TFBG plotted against three kinds of liquids. (a) Cladding mode, (b) core mode. Solid line: water, broken line: glycerine $24 \%$, dotted line: glycerine $35 \%$.

In contrast, no spectral change of the core mode at $1571.56 \mathrm{~nm}$ was observed with the change in the refractive index, as shown in Fig. $4 b$.

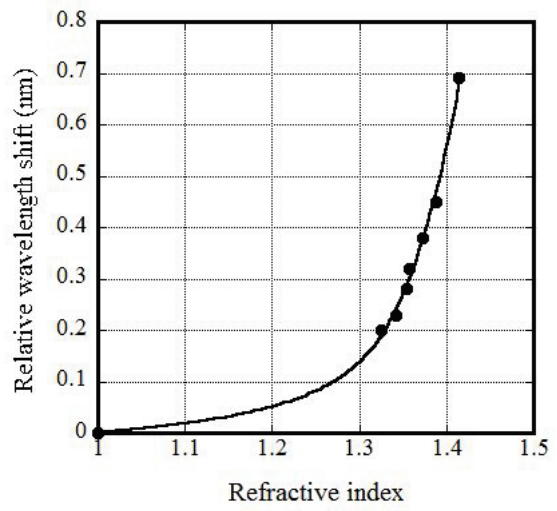

Fig. 5 Relative wavelength shift of $7.3^{\circ}$-TFBG as a function of the refractive index of liquids and air.

Figure 5 shows the relative wavelength shift plotted as a function of the refractive index $n_{l}$ of the liquids and air for a $7.3^{\circ}$-TFBG. The filled circles indicate the wavelength shifts from the wavelength in air, and the solid line is the fitting curve $f\left(n_{l}\right)$ expressed as a function of the refractive index $n_{l}$ of liquids and air. The line increased exponentially for the index and is expressed by the following equation,

$$
f\left(n_{l}\right)=A\left\{e^{B\left(n_{l}-l\right)}-1\right\}
$$

where $A$ and $B$ are experimental parameters. As shown in the figure, the wavelength shifted to $0.68 \mathrm{~nm}$ for the $7.3^{\circ}$ TFBG, which corresponds to the index from 1.00 to 1.41 .

\subsection{Characteristics of peak shift against temperature of liquid}

Figures $6 \mathrm{a}$ and $6 \mathrm{~b}$ show the transmission spectra of the $7.3^{\circ}$-TFBG in water at 293,313 and $353 \mathrm{~K}$. As shown in Fig. 6a, the peak wavelength of the 1540-nm- cladding mode became longer as the temperature of liquid increased, and the wavelength shifted from $1540.68 \mathrm{~nm}$ to 1541.16 $\mathrm{nm}$, which correspond to the temperature changes from 293 $\mathrm{K}$ to $353 \mathrm{~K}$. In contrast, as shown in Fig. 6b, the peak wavelength of the core mode shifted from $1571.56 \mathrm{~nm}$ to $1572.04 \mathrm{~nm}$.
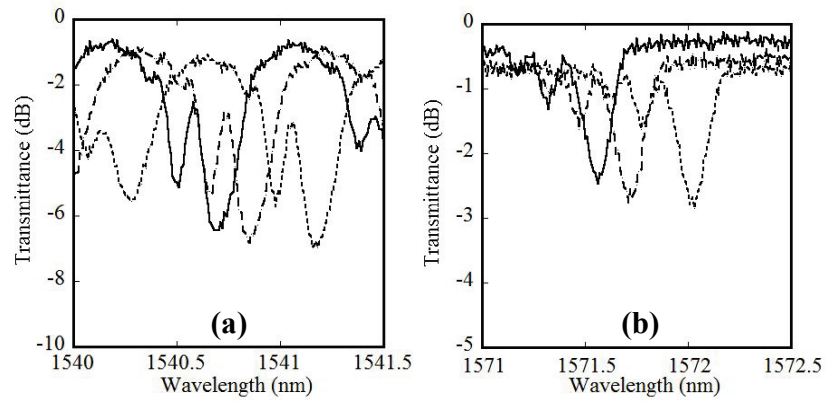

Fig. 6 Peak shift of core and cladding modes of $7.3^{\circ}$-TFBG plotted against the temperature of liquids. (a) Cladding mode, (b) core mode. Solid line: $293 \mathrm{~K}$, broken line: $313 \mathrm{~K}$, dotted line: $353 \mathrm{~K}$.

Figure 7 shows the relative wavelength shift of the core and three selected cladding modes plotted against a function of the temperature of the liquid for the $7.3^{\circ}$-TFBG. For comparison, we selected three peaks of the cladding mode at 1560,1540 , and $1525-\mathrm{nm}$ that were respectively located on the longer wavelength side, near the middle, and on the shorter side. The inset shows the relative wavelength shift for the range from $335 \mathrm{~K}$ to $360 \mathrm{~K}$. The straight lines in the figure are the fitting curve $g\left(T_{l}\right)$ as a function of the temperature $T_{l}$ of water. The lines increased linearly with the temperature and are expressed by the following equation,

$$
g\left(T_{l}\right)=C\left(T_{l}-T_{0}\right)
$$

where $T_{0}$ and $C$ are the reference temperature (e.g. $293 \mathrm{~K}$ ) of water and an experimental parameter. The wavelength of the core mode (open circles and solid line) shifted to 0.48 $\mathrm{nm}$, while the temperature increased from $293 \mathrm{~K}$ to $353 \mathrm{~K}$. The wavelengths of the 1560-nm (open squares and dashed line), 1540-nm (open rhomboids and broken line), and 1525-nm (open triangles and dotted line) cladding modes shifted to $0.53,0.48$, and $0.47 \mathrm{~nm}$, respectively. The three plot symbols and lines representing the core mode and the $1525-\mathrm{nm}$ and 1540-nm cladding modes almost overlap in the figure. As shown in the inset, the peak wavelength shift of the 1540-nm cladding mode is almost the same as that of the core mode.

On the basis of our experimental results, we can say that the TFBG was successfully fabricated by combining the 266-nm laser and the phase mask. This technique is 
simpler than the conventional method that uses a 244-nm laser and a Lloyd mirror interferometer [6].

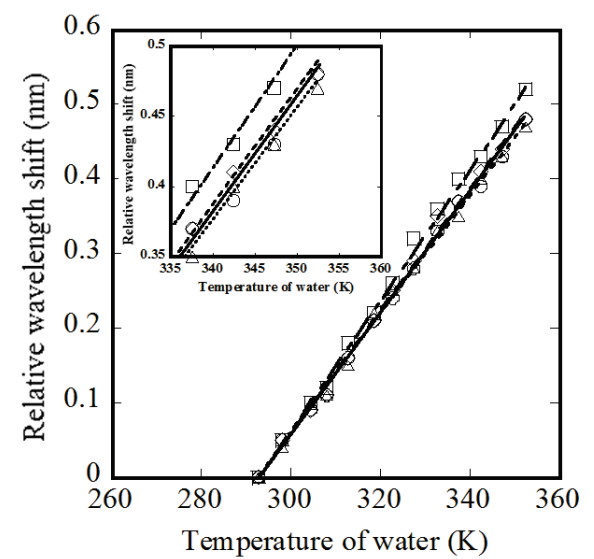

Fig. 7 Relative wavelength shift of cladding mode of $7.3^{\circ}$ TFBG as a function of the temperature of water. $\bigcirc$ : core mode, $\square: 1560$-nm cladding mode, $\diamond: 1540$ $\mathrm{nm}$ cladding mode, $\triangle: 1525$-nm cladding mode.

\section{Discussion}

In this section, we discuss the dependence of the peak wavelength shifts on the refractive index and temperature of liquids. The peak wavelength of the cladding mode increased exponentially as the refractive index increased, although that of the core mode did not change. On the other hand, the wavelength of both the core and cladding modes increased linearly as the temperature increased. We would like to point out that the behavior of the peak of cladding modes was different from that of the core mode. Utilizing these facts, we were able to separate the effect of the refractive index and the temperature on the wavelength shift. We can express the peak wavelength shift of the cladding mode by the following equation,

$$
h\left(n_{l}, T_{l}\right)=A\left\{e^{B\left(n_{l}-1\right)}-1\right\}+C\left(T_{l}-T_{0}\right)
$$

where $n_{l}$ and $T_{l}$ are the index and the temperature of liquids, respectively. Furthermore, we can express the peak wavelength shift of the core mode by the following equation,

$$
g^{\prime}\left(n_{l}, T_{l}\right)=C^{\prime}\left(T_{l}-T_{0}\right)
$$

We also point out that the peak wavelength shift of the lower-order mode (longer wavelength side) is larger than that of the higher-order mode (shorter wavelength side), as shown in Fig. 7. It is clear that the peak wavelength shift per degree of at least one cladding mode is the same as that of the core mode, although the peak wavelength shift of each cladding mode is different from each other.
Therefore, we can measure the refractive index and temperature of liquids simultaneously by using the peak wavelength shifts of the core and cladding modes.

Finally, we also discuss the range of temperatures that can be measured with this technique. The wavelength between one peak and the neighboring peak of the cladding mode at the $1540-\mathrm{nm}$ peak was approximately $1.3 \mathrm{~nm}$. The peak shifted to $0.008 \mathrm{~nm}$ per $1 \mathrm{~K}$. Therefore, we can measure it from $130 \mathrm{~K}$ to $450 \mathrm{~K}$ but cannot measure it when the liquid freezes, because a gap occurs between the TFBG and a solid. This temperature range is sufficient for measuring bio-chemical phenomena in the medical and biological fields.

\section{Conclusion}

We fabricated a TFBG by using a simple technique combining a 266-nm laser and a phase mask. This technique makes it easy to adjust the optical alignment. Furthermore, use of the 266-nm laser advantageous when considering this technique for commercial applications.

We confirmed that the $7.3^{\circ}$-TFBG fabricated using this technique was able to simultaneously measure the refractive index and the temperature of liquid. We found that a refractive index from 1 to 1.41 and a temperature from 293 to $353 \mathrm{~K}$ could be measured with a single probe of the fabricated TFBG. It was identifiable from a difference in the refractive index of only 0.0148 . TFBGs are considered to be very promising for use as sensors of bio-chemical phenomena in the medical and biological fields.

\section{References}

[1] R. Kashyap: "Fiber Bragg Gratings" (Academic Press, San Diego, 1999) p.12. (Books)

[2] X. Dong, H. Zhang, B. Liu and Y. Miao: Photonic Sensors, 1, (2011) 6.(Journals)

[3] Y. Liu, Q. Liu, and K. S. Chiang: Opt. Lett., 34, (2009) 1726.(Journals)

[4] A. Othonos and K. Kalli: "Fiber Bragg Gratings: Fundamentals and Applications in Telecommunication and Sensing"', (Artech House, Boston, 1999) p.1. (Books)

[5] T. Erdogan: J. Opt. Soc. Am. A, 14, (1997) 1760. (Journals)

[6] G. Laffont and G. Ferdinand: Meas. Sci. Technol., 12, (2001) 765. (Journals)

[7] A. Kameyama, E. Maurana, M. Katto and A. Yokotani: The Review of Laser Eng., 40, (2012) 883. (Journals)

[8] J. Rheims: Meas. Sci. Technol., 8, (1997) 601. (Journals)

(Received: March 31, 2014, Accepted: August 27, 2014) 